\title{
Correction: The role of cumulative physical work load in symptomatic knee osteoarthritis - a case-control study in Germany (Seidler et al. 2008).
}

Andreas Seidler ${ }^{1 *}$, Ulrich Bolm-Audorff ${ }^{2}$, Nasreddin Abolmaali ${ }^{3}$ and Gine Elsner ${ }^{4}$

\section{Correction}

In the original paper [1], there is a mistake in the results of the occupational group analysis. This mistake occurred when the core data set was merged with the occupational group data. According to the modified occupational group analysis (see modified Table 1), OR for chemical processers and manufacturers of plastics products are no longer significantly elevated. Having worked more than 10 years as metal worker is associated with knee osteoarthritis ( $\mathrm{OR}=2.2 ; 95 \%$ CI 1.1-4.4). The knee osteoarthritis risk of plasterers, insulators, glaziers, terrazzo workers, construction carpenters, roofers, and upholsters approaches statistical significance in the long-duration category $(\mathrm{OR}=3.7 ; 95 \mathrm{CI}$ 0.9-15.2). For woodworkers, the knee osteoarthritis risk is no longer significantly elevated. Having worked more than 10 years as painter or varnisher is associated with knee osteoarthritis $(\mathrm{OR}=9.6$; $95 \%$ CI 1.2-77.9). Finally, we find a significantly elevated OR of 3.2 (95\% CI 1.1-9.1) among subjects having worked as physically exposed service workers (storemen, nurses, refuse collectors) for more than 10 years. When subjects with non-service work as main occupation ("blue-collar

Table 1 Occupational groups (reference group: service occupation as main occupation) and symptomatic knee osteoarthritis

\begin{tabular}{|c|c|c|c|c|c|c|c|c|c|c|c|c|}
\hline \multirow[t]{2}{*}{ Specific occupational groups ${ }^{a}$} & \multicolumn{6}{|c|}{1 to 10 yrs. in specific occ. group } & \multicolumn{6}{|c|}{$>10$ yrs. in specific occ. group } \\
\hline & Cases & $\%$ & Controls & $\%$ & Adj. $\mathrm{OR}^{\mathrm{b}}$ & $95 \% \mathrm{Cl}$ & Cases & $\%$ & Controls & $\%$ & Adj. $\mathrm{OR}^{\mathrm{b}}$ & $95 \% \mathrm{Cl}$ \\
\hline \multicolumn{13}{|l|}{ Agriculture and mining } \\
\hline $\begin{array}{l}\text { Agricultural, animal husbandry, and } \\
\text { forestry workers }\end{array}$ & 10 & 3.4 & 12 & 3.7 & 1.6 & $0.5-4.6$ & 6 & 2.0 & 2 & 0.6 & 1.6 & $0.3-8.5$ \\
\hline \multicolumn{13}{|l|}{ Production } \\
\hline $\begin{array}{l}\text { Chemical processers and manufacturers } \\
\text { of plastics product }\end{array}$ & 6 & 2.0 & 7 & 2.1 & 0.9 & $0.2-3.4$ & 12 & 4.1 & 5 & 1.5 & 1.8 & $0.5-6.5$ \\
\hline $\begin{array}{l}\text { Manufacturers of paper and paper } \\
\text { products; printers }\end{array}$ & 1 & 0.3 & 3 & 0.9 & - & - & 10 & 3.4 & 5 & 1.5 & 1.7 & $0.5-5.6$ \\
\hline Metal processers, blacksmiths & 11 & 3.7 & 1 & 0.3 & 14.6 & $1.5-142$ & 10 & 3.4 & - & - & - & - \\
\hline $\begin{array}{l}\text { Metal workers (machinery fitters, } \\
\text { machine assemblers, mechanics, } \\
\text { manufacturers of precision instruments; } \\
\text { plumbers, welders, sheet metal and } \\
\text { structural metal preparers and erectors) }\end{array}$ & 28 & 9.5 & 42 & 12.8 & 0.9 & $0.5-1.8$ & 45 & 15.3 & 19 & 5.8 & 2.2 & $1.1-4.4$ \\
\hline Electrical and electronics workers & 4 & 1.4 & 18 & 5.5 & 0.2 & $0.05-0.7$ & 13 & 4.4 & 11 & 3.4 & 1.6 & $0.6-4.3$ \\
\hline Tanners, fellmongers, pelt dressers; & 4 & 1.4 & 2 & 0.6 & 1.2 & $0.2-7.5$ & 3 & 1.0 & 2 & 0.6 & 1.2 & $0.2-8.1$ \\
\hline
\end{tabular}

\footnotetext{
* Correspondence: andreas.seidler@mailbox.tu-dresden.de

${ }^{1}$ Institute and Policlinic for Occupational and Social Medicine, Dresden,

Germany

Full list of author information is available at the end of the article
} 
Table 1 Occupational groups (reference group: service occupation as main occupation) and symptomatic knee osteoarthritis (Continued)

\begin{tabular}{|c|c|c|c|c|c|c|c|c|c|c|c|c|}
\hline $\begin{array}{l}\text { Food and beverage processors; tobacco } \\
\text { product makers }\end{array}$ & 8 & 2.7 & 10 & 3.1 & 1.5 & $0.4-5.3$ & 10 & 3.4 & 8 & 2.4 & 1.4 & $0.4-4.9$ \\
\hline $\begin{array}{l}\text { Construction workers (structural } \\
\text { engineering, civil engineering) }\end{array}$ & 14 & 4.7 & 9 & 2.8 & 2.3 & $0.7-6.9$ & 10 & 3.4 & 3 & 0.9 & 1.7 & $0.4-7.1$ \\
\hline $\begin{array}{l}\text { Plasterers, insulators, glaziers, terazzo } \\
\text { workers, construction carpenters, roofers; } \\
\text { upholsterers }\end{array}$ & 6 & 2.0 & 7 & 2.1 & 0.6 & $0.2-2.4$ & 10 & 3.4 & 4 & 1.2 & 3.7 & $0.9-15.2$ \\
\hline $\begin{array}{l}\text { Woodworkers and plastic workers } \\
\text { (carpenters, cabinet makers, wooden or } \\
\text { plastic models makers, wood-frame } \\
\text { construction) }\end{array}$ & 10 & 3.4 & 5 & 1.5 & 2.3 & $0.6-8.1$ & 7 & 2.4 & 3 & 0.9 & 3.3 & $0.7-16.0$ \\
\hline Painters; varnishers & 4 & 1.4 & 7 & 2.1 & 1.3 & $0.3-6.3$ & 12 & 4.1 & 1 & 0.3 & 9.6 & $1.2-77.9$ \\
\hline Quality inspectors; packers & 10 & 3.4 & 1 & 0.3 & 19.7 & $2.0-190$ & 3 & 1.0 & 2 & 0.6 & 2.5 & $0.2-31.6$ \\
\hline Labourers & 7 & 2.4 & 9 & 2.8 & 2.7 & $0.8-9.1$ & - & - & - & - & - & - \\
\hline $\begin{array}{l}\text { Operators (crane and earth-moving } \\
\text { machinery operators etc.) }\end{array}$ & 2 & 0.7 & 3 & 0.9 & 0.4 & $0.04-3.5$ & 1 & 0.3 & 2 & 0.6 & - & - \\
\hline \multicolumn{13}{|l|}{ Technology } \\
\hline $\begin{array}{l}\text { Technicians (engineers, architects, } \\
\text { chemists, physicists, electrical } \\
\text { engineering technicians) }\end{array}$ & 11 & 3.7 & 24 & 7.3 & 0.7 & $0.3-1.7$ & 41 & 13.9 & 32 & 9.8 & 1.3 & $0.7-2.4$ \\
\hline \multicolumn{13}{|l|}{ Services } \\
\hline $\begin{array}{l}\text { Service workers: Storemen, nurses, } \\
\text { refuse collectors }\end{array}$ & 16 & 5.4 & 19 & 5.8 & 1.3 & $0.6-3.0$ & 16 & 5.4 & 8 & 2.4 & 3.2 & $1.1-9.1$ \\
\hline Soldiers & 3 & 1.0 & 4 & 1.2 & 0.4 & $0.04-3.1$ & 1 & 0.3 & 1 & 0.3 & - & - \\
\hline Other service workers & 1 & 0.3 & 5 & 1.5 & 0.5 & $0.1-5.8$ & - & - & 1 & 0.3 & - & - \\
\hline
\end{tabular}

a Occupations with $<10$ subjects are not shown.

${ }^{b}$ Adjusted for age, region, body-mass index, and jogging/athletics.

workers") are compared with "white-collar workers", the odds ratio for knee osteoarthritis is still significantly elevated $(\mathrm{OR}=2.0 ; 95 \%$ CI 1.3-2.9).

\section{Author details}

'Institute and Policlinic for Occupational and Social Medicine, Dresden, Germany. ${ }^{2}$ Division of Occupational Health, RP Darmstadt, Wiesbaden, Germany. ${ }^{3}$ OncoRay - Ml OncoRay, Dresden, Germany. Institute of Occupational Medicine, Johann Wolfgang Goethe-University, Frankfurt am Main, Germany.

Received: 25 September 2012 Accepted: 26 September 2012 Published: 10 October 2012

\section{Reference}

1. Seidler A, Bolm-Audorff U, Abolmaali N, Elsner G, Knee osteoarthritis study group: The role of cumulative physical work load in symptomatic knee osteoarthritis - a case-control study in Germany. J Occup Med Toxicol 2008, 3:14.

doi:10.1186/1745-6673-7-21

Cite this article as: Seidler et al:: Correction: The role of cumulative physical work load in symptomatic knee osteoarthritis - a case-control study in Germany (Seidler et al. 2008). Journal of Occupational Medicine and Toxicology 2012 7:21.

\section{Submit your next manuscript to BioMed Central and take full advantage of:}

- Convenient online submission

- Thorough peer review

- No space constraints or color figure charges

- Immediate publication on acceptance

- Inclusion in PubMed, CAS, Scopus and Google Scholar

- Research which is freely available for redistribution 